\title{
The $5000 \%$ case: a glimpse into the financial issue of lung cancer treatment
}

\author{
Jordi Remon ${ }^{1}$, Julia Bonastre ${ }^{2,3}$ and Benjamin Besse $e^{1,4}$ \\ Affiliations: ${ }^{1}$ Medical Oncology Dept, Institute Gustave Roussy, Villejuif, France. ${ }^{2}$ Service de biostatistique et \\ d'épidémiologie, Institute Gustave Roussy, Villejuif, France. ${ }^{3}$ CESP Centre for Research in Epidemiology and \\ Population Health, INSERM U1018, Université Paris-Sud, UVSQ, Université Paris-Saclay, Villejuif, France. \\ ${ }^{4}$ Université Paris-Sud and Institute Gustave Roussy Cancer Campus, Villejuif, France.
}

Correspondence: Benjamin Besse, Medical Oncology Dept, Institute Gustave Roussy, 117 rue E. Vaillant, Villejuif 95805, France. E-mail: Benjamin.BESSEQgustaveroussy.fr

\section{-} $@$ ERSpublications

Discrepancies in access to personalised treatment could be due to access to molecular testing and treatment costs http://ow.ly/ZxK5v

A critical issue concerning drug treatment costs was recently brought, somewhat dramatically, into the public arena accompanied by heated political debate after an unexpected overnight increase in the price of Daraprim, the only approved treatment for toxoplasmosis, which has been available for over 60 years. In August 2015, Turing Pharmaceuticals (New York, NY, USA), a start-up founded by a former hedge fund manager, acquired the drug and immediately raised the price from $\$ 13.50$ a tablet to $\$ 750$ [1]. For many oncologists, this unaffordable price echoed the impressive costs of many recently released cancer drugs. A new end-point in the development of cancer drugs has recently been put forward, taking this financial parameter into consideration. The term "financial toxicity" has been coined, reflecting the patient impact of the costs of cancer due to out-of-pocket expenses (i.e. costs not covered by government or private insurance) [2].

Lung cancer is the leading cause of cancer-related deaths worldwide [3]. Recently, nonsmall cell lung cancer (NSCLC) was divided into several subsets based on molecular profiles that may match targeted therapies [4]. In around 15\% of Caucasian patients, management of this disease has been revolutionised in the past 10 years, and standard of care is upfront-targeted therapy. This personalised medicine approach has a large impact on patient survival [5], supporting the clinical benefit of this policy [4]. Mutations in the Epidermal Growth Factor Receptor $(E G F R)$ gene $[6,7]$ are an example of relevant predictive biomarkers for response to EGFR tyrosine kinase inhibitors (TKIs) [8]. High rates of efficacy have been achieved thanks not only to the targeted therapy approach, but also to intensive drug development, with the approval of five drugs in the space of less than 7 years for advanced EGFR-mutant NSCLC, namely erlotinib, gefitinib, afatinib and icotinib (in first-line setting) and osimertinib (in second-line setting).

In the present issue of European Respiratory Journal, CARBonnaux et al. [9] highlight two major financial issues, namely, as expected, cost in terms of access to the drug but primarily the cost in terms of access to molecular testing. Almost 10 years after the identification of the EGFR mutation [6,7], inequalities in the availability of the EGFR mutation test exist worldwide. CARBonnaux et al. [9] have reported that EGFR mutational analysis was available for $70 \%$ of the world's population. Availability of the analysis, its cost and the delay for obtaining the results significantly correlated with the country's Human Development Index (HDI). However, as the authors report, their study design (cross-over expert opinion survey) may have biased outcome considering that most of the non-responding countries had low HDI ranks. However, as the test is unlikely to be routinely available in these countries, the final results of the study are therefore unlikely to be underestimated. The authors reported that when performed, results of EGFR mutation status were available within 7 days in $26 \%$ of the worldwide population, mostly in countries with a high HDI. Given the superior outcome in several randomised phase III trials with EGFR TKI compared with

Received: March 162016 | Accepted: March 162016

Conflict of interest: Disclosures can be found alongside the online version of this article at erj.ersjournals.com

Copyright @ERS 2016 
first-line chemotherapy, their indication as upfront treatment has been recognised in most of the guidelines [10]. In some of these phase III clinical trials, approximately one-third of patients with the EGFR mutation who were randomly assigned to first-line chemotherapy could not receive EGFR TKI as salvage therapy, implying a strong detriment to patient survival [11-14] and reinforcing that a delay in mutational analysis results might have a critical impact on personalised treatment.

In the global EGFR MutMap II systematic review, the rate of EGFR mutation was higher in the Asia-Pacific subgroup at $47 \%$ compared to $15 \%$ in the European subgroup [15]. In all regional subgroups, EGFR mutation frequency was higher in women compared with men, and in never-smokers compared with ever-smokers $[15,16]$. This study reiterates the well-known clinicopathological features correlating with EGFR mutation (in particular East Asian ethnicity and lack of smoking history). Nonetheless, it lacks data from several large geographic regions, notably Africa, the Middle East, Central Asia and South America. However, in a recent study, 50\% of Asian patients with EGFR mutations were not female non-smokers [17]. Interestingly, CARBONNAUX et al. [9] reported higher EGFR mutation positivity in Hispanic populations (30\% in Mexico and 25\% in Colombia) compared with Caucasian population $(\sim 11 \%)$ [4]. Of note, although tobacco consumption has been related to molecular abnormalities (such as KRAS or STK11 mutations [18, 19]), there are no aetiological findings that explain the occurrence of somatic EGFR mutations. It has also been reported that passive smoking has no impact on the somatic profile of lung cancer in never-smokers suggesting other aetiological factors [20]. Among the potential causes, environmental exposures such as radon [21] or virus could be explored, with a recent suggestion that in the Chinese population (with a 50\% rate of EGFR mutant adenocarcinoma) Merkel cell polyomavirus infection might have a role in the carcinogenesis in this NSCLC subtype [22]. Research into the epidemiological causes of the different proportions of EGFR mutations worldwide is awaited.

In the European Union, the cost of cancer therapy in 2009 was $€ 126$ billion (equivalent to $€ 102$ per citizen, with wide differences by country). Among them, lung cancer had the highest economic cost ( $€ 18.8$ billion) amounting to $15 \%$ of the overall cancer costs followed by breast cancer (12\%), colorectal cancer (10\%) and prostate cancer (7\%). Drug expenditure accounted for $\sim 27 \%$ of cancer-related healthcare costs [23]. The heterogeneity of health insurance systems in European countries or in countries with the highest HDI is likely to explain (as reported by CARBOnNaux et al. [9]) the difference in patient access to the main EGFR TKI (erlotinib, gefitinib and afatinib) and the variation of treatment costs between countries. The cost per patient for new anticancer drugs reaches $\$ 100,000$ or more annually [24]. Although some studies suggest that EGFR TKI are cost-effective compared to standard first-line chemotherapy (cisplatin-pemetrexed) in advanced NSCLC patients [25], treatment costs are high. CARBonnaux et al. [9] reported a mean monthly out-of-pocket expense for these treatments of $\$ 500-\$ 1000$ per patient worldwide and it was even higher in the USA, reaching $\$ 1500$. Moreover, contrary to chemotherapy that is prescribed for four to six cycles, EGFR TKIs are prescribed until disease progression.

It is well known that the costs of drug development are high and that only a small portion of drugs in the clinical development pipeline ultimately reach the market. A recent study reported that the total cost for developing a new drug was $\$ 2.6$ billion. However, nearly half that total cost ( $\$ 1.2$ billion) was ascribed to the cost of the capital, with only $\$ 1.4$ billion attributed to funds actually spent on research [26]. In the case of highly potent innovate drugs, such as EGFR TKIs, the sales benefit is likely to rapidly cover the cost of the investment. For example, the total funds invested in the development for sofosbuvir (Sovaldi; Gilead Sciences Inc., Foster City, CA, USA), a drug against hepatitis C, were recovered during its first year of sales after approval in late 2013 (based on $\$ 1000$ per tablet) [27]. However, the treatment is unaffordable for most healthcare systems. This dramatically questions the access to highly innovative drugs [27]. Although one might expect a decrease in cost with time due to the commercialisation of the drugs, generics and biosimilars [28], ironically, total expenditure for anticancer drugs frequently increases. As an example, when imatinib, a pioneer drug in chronic myeloid leukaemia that dramatically increased patient survival, was introduced in 2001 the cost was roughly $\$ 30,000$ per year of treatment in the USA. By 2012 , this had more than tripled to $\$ 92,000$ [29]. The out-of-pocket cost was analysed between 2001 and 2011 and a clear link was established between high patient co-payment requirements and low adherence to imatinib. These data suggest that it is important to develop rational policies that do not inhibit patient's access to highly effective life-extending treatments [29].

Unfortunately, the large discrepancies in access to personalised medicine in lung cancer patients between countries presented by CARBOnNaux et al. [9] are only likely to increase. The price of the promising therapeutic approach with immunotherapy has reached a new level in treatment costs. These data contribute to public health and policy intelligence, which is required to deliver affordable cancer care systems and inform effective allocation of public research funds. The words of SidDiQUi and RaJKUmar [24] are valid more than ever, "Ultimately, we as a society must find a balance between health care affordability and profits that will provide the necessary incentive for continued innovation. Not doing so risks creating a health care system in which all participants lose". 


\section{References}

1 Center for Global Development. The 5000\% price increase and the economic case for pharma price regulation. www.cgdev.org/blog/5000-price-increase-and-economic-case-pharma-price-regulation Date last updated: September 23, 2015. Date last accessed: March 14, 2016.

2 Zafar SY, Peppercorn JM, Schrag D, et al. The financial toxicity of cancer treatment: a pilot study assessing out-of-pocket expenses and the insured cancer patient's experience. Oncologist 2013; 18: 381-390.

3 Ferlay J, Soerjomataram I, Dikshit R, et al. Cancer incidence and mortality worldwide: sources, methods and major patterns in GLOBOCAN 2012. Int J Cancer 2015; 136: E359-E386.

4 Barlesi F, Mazieres J, Merlio J-P, et al. Routine molecular profiling of patients with advanced non-small-cell lung cancer: results of a 1-year nationwide programme of the French Cooperative Thoracic Intergroup (IFCT). Lancet 2016; [in press DOI: 10.1016/S0140-6736(16)00004-0].

5 Kris MG, Johnson BE, Berry LD, et al. Using multiplexed assays of oncogenic drivers in lung cancers to select targeted drugs. JAMA 2014; 311: 1998-2006.

6 Lynch TJ, Bell DW, Sordella R, et al. Activating mutations in the epidermal growth factor receptor underlying responsiveness of non-small-cell lung cancer to gefitinib. N Engl J Med 2004; 350: 2129-2139.

7 Kobayashi S, Boggon TJ, Dayaram T, et al. EGFR mutation and resistance of non-small-cell lung cancer to gefitinib. N Engl J Med 2005; 352: 786-792.

8 Sebastian M, Schmittel A, Reck M. First-line treatment of EGFR-mutated nonsmall cell lung cancer: critical review on study methodology. Eur Respir Rev 2014; 23: 92-105.

9 Carbonnaux M, Souquet P-J, Meert A-P, et al. Inequalities in lung cancer: a world of EGFR. Eur Respir J 2016; 47: 1502-1509.

10 Besse B, Adjei A, Baas P, et al. 2nd ESMO Consensus Conference on Lung Cancer: non-small-cell lung cancer first-line/second and further lines of treatment in advanced disease. Ann Oncology 2014; 25: 1475-1484.

11 Mitsudomi T, Morita S, Yatabe Y, et al. Gefitinib versus cisplatin plus docetaxel in patients with non-small-cell lung cancer harbouring mutations of the epidermal growth factor receptor (WJTOG3405): an open label, randomised phase 3 trial. Lancet Oncol 2010; 11: 121-128.

12 Zhou C, Wu YL, Chen G, et al. Final overall survival results from a randomised, phase III study of erlotinib versus chemotherapy as first-line treatment of EGFR mutation-positive advanced non-small-cell lung cancer (OPTIMAL, CTONG-0802). Ann Oncol 2015; 26: 1877-1883.

13 Rosell R, Carcereny E, Gervais R, et al. Erlotinib versus standard chemotherapy as first-line treatment for European patients with advanced EGFR mutation-positive non-small-cell lung cancer (EURTAC): a multicentre, open-label, randomised phase 3 trial. Lancet Oncol 2012; 13: 239-246.

14 Mok TS, Wu Y-L, Thongprasert S, et al. Gefitinib or carboplatin-paclitaxel in pulmonary adenocarcinoma. $N$ Engl J Med 2009; 361: 947-957.

15 Midha A, Dearden S, McCormack R. EGFR mutation incidence in non-small-cell lung cancer of adenocarcinoma histology: a systematic review and global map by ethnicity (mutMapII). Am J Cancer Res 2015; 5: 2892-2911.

16 Pao W, Chmielecki J. Rational, biologically based treatment of EGFR-mutant non-small-cell lung cancer. Nat Rev Cancer 2010; 10: 760-774.

17 Shi Y, Au JS-K, Thongprasert S, et al. A prospective, molecular epidemiology study of EGFR mutations in Asian patients with advanced non-small-cell lung cancer of adenocarcinoma histology (PIONEER). J Thorac Oncol 2014; 9: $154-162$.

18 Dogan S, Shen R, Ang DC, et al. Molecular epidemiology of EGFR and KRAS mutations in 3,026 lung adenocarcinomas: higher susceptibility of women to smoking-related KRAS-mutant cancers. Clin Cancer Res 2012; 18: 6169-6177.

19 Kempf E, Rousseau B, Besse B, et al. KRAS oncogene in lung cancer: focus on molecularly driven clinical trials. Eur Respir Rev 2016; 25: 71-76.

20 Couraud S, Debieuvre D, Moreau L, et al. No impact of passive smoke on the somatic profile of lung cancers in never-smokers. Eur Respir J 2015; 45: 1415-1425.

21 Torres-Durán M, Ruano-Ravina A, Parente-Lamelas I, et al. Lung cancer in never-smokers: a case-control study in a radon-prone area (Galicia, Spain). Eur Respir J 2014; 44: 994-1001.

$22 \mathrm{Xu} \mathrm{S}$, Jiang J, Yu X, et al. Association of Merkel cell polyomavirus infection with EGFR mutation status in Chinese non-small cell lung cancer patients. Lung Cancer 2014; 83: 341-346.

23 Luengo-Fernandez R, Leal J, Gray A, et al. Economic burden of cancer across the European Union: a population-based cost analysis. Lancet Oncol 2013; 14: 1165-1174.

24 Siddiqui M, Rajkumar SV. The high cost of cancer drugs and what we can do about it. Mayo Clin Proc 2012; 87: 935-943.

25 Vergnenegre A, Massuti B, de Marinis F, et al. Economical analysis of first line treatment by erlotinib in an EGFR mutated population with advanced non-small cell lung cancers NSCLC. J Thorac Oncol 2016; [in press DOI: 10.1016/j.jtho.2016.02.004].

26 Avorn J. The \$2.6 billion pill - methodologic and policy considerations. N Engl J Med 2015; 372: 1877-1879.

27 Liu S, Watcha D, Holodniy M, et al. Sofosbuvir-based treatment regimens for chronic, genotype 1 hepatitis C virus infection in U.S. incarcerated populations: a cost-effectiveness analysis. Ann Intern Med 2014; 161: 546-553.

28 Bach PB. Limits on Medicare's ability to control rising spending on cancer drugs. N Engl J Med 2009; 360: 626-633.

29 Dusetzina SB, Winn AN, Abel GA, et al. Cost sharing and adherence to tyrosine kinase inhibitors for patients with chronic myeloid leukemia. J Clin Oncol 2014; 32: 306-311. 